\title{
Labeling, Rehearsal, and Short-Term Memory in Retarded Children ${ }^{1}$
}

\author{
John W. Hagen, Lynn A. Streeter, and Richard Raker \\ The University of Michigan
}

\begin{abstract}
A short-term memory task was used to explore the effects of verbal labeling and rehearsal on serial-position recall in mildly retarded 9- to 11year-old children. A stimulus array consisting of seven cards depicting familiar animals was presented for seven trials. In Expt I, recall when subjects labeled the pictures as they were shown was compared to recall when no labeling occurred. Total recall was not affected, but for the older CA group primacy recall was hindered and recency recall was facilitated by labeling. In Expt II, three variations of rehearsal of the names to be recalled were compared. When prompting accompanied rehearsal, recall improved at both recency and primacy positions. When prompting occurred for the primacy positions only, recall was higher for these positions but not for other positions. These results support the view that verbal skills affect recall in mildly retarded children similarly to normal children.
\end{abstract}

The relation of verbal skills to short-term memory in children recently has been the subject of considerable research and discussion (Flavell, 1970, 1971). Labeling of to-be-remembered information has been found to facilitate recall at certain age levels but not others (Hagen \& Kingsley, 1968; Kenney, Cannizzo \& Flavell, 1967). Evidence that retardates are deficient in short-term memory has been available for some time (Ellis, 1963), but only recently have there been systematic altempts to account for the deficiency (Ellis, 1970). Ellis found that normal subjects' recall of initial items in a serial recall list was facilitated when the time interval between presentation of stimuli was increased, but no improvement was found in primacy recall for retardates when time was increased. These findings were interpreted as indicating that retardates do not use verbal rehearsal as a strategy to facilitate recall as do normal subjects. Rehearsal in memory in retarded adolescents and adults has been explored by Belmont and Butterfield (1971). Thus far, however, there has been

\footnotetext{
${ }^{1}$ This research was supported in part by Grant No. 317300 from the Horace $H$. Rackham School of Graduate Studies, University of Michigan. The authors wish to thank the superintendent, Dr. P. Buoniconto, and staff of the Wayne County Child Development Center, Northville MI, for their gracious cooperation during the various phases of this research project. Author Hagen's address: Department of Psychology, University of Michigan, Ann Arbor, MI 48104.
} 
little research on the role of verbal skills and memory in retarded children.

The two experiments reported here were performed to determine whether supplying the retarded child with either labels or rehearsal strategies that facilitated the performance of normal children within the same MA range (Hagen \& Kingsley, 1968; Kingsley \& Hagen, 1969) would similarly affect retardates' performance. If retardates are found to perform similarly to normal children of equivalent MA levels in terms of developmental changes, and are able to benefit from induced rehearsal, it would support the view that, rather than a short-term memory deficit, a developmental lag characterizes their performance.

The serial position short-term memory task used in previous studies was used here (Hagen \& Kingsley, 1968; Hagen, Meacham \& Mesibov, 1970). Half the subjects at each age level were required to say the names of the pictures to be remembered and half were not. In one study (Hagen \& Kingsley, 1968), labeling did not affect recall at CA 4 years but it facilitated recall at ages 6 and 8 years. At age 10 years, recall was higher than at any of the lower ages, but labeling did not affect it. The serial-position analyses proved revealing. At the recency positions (the positions occupied by those pictures shown just before recall was measured) recall was high at all ages and was facilitated by labeling. However, at the primacy positions (those at the beginning of the serial list) there was a developmental trend; recall was low at the youngest $\mathrm{CA}$ level but improved at the older CA levels. At the older CA levels primacy recall was lower when labeling of the stimuli was required than when it was not. At CA 10 years, the improved recall at the recency portion of the curve was canceled out by the decrement at the primacy portion. Similar findings occurred at ages 12 and 14 years in a subsequent study (Hagen, Meacham \& Mesibov, 1970).

Another study (Kingsley \& Hagen, 1969) was performed to determine if young children could be induced to usc vcrbal rehearsal in the memory task. It had been observed in the earlier studies that older, although not the younger, subjects often said aloud the names of the to-he-remembered stimuli in serial order during presentation of the stimuli. When overt labeling was required, it may have interfered with this spontaneous rehearsal. In Kingsley and Hagen's study, 5-year-old subjects were required to repeat aloud the names of the pictures in serial order. As each new picture appeared, its name was added to the series of spoken names. Recall was better when this verbal rehearsal technique was used, compared to both a control condition with no rehearsal and when overt labeling was required. Moreover, the facilitation occurred mainly at the primacy portion of the recall curve, where performance 
of subjects of this age level had been found to be low in the earlier study.

It was hypothesized that retarded children's short-term memory would be affected by the same manipulations of verbal performance found to affect memory in normal children, i.e., naming and overt serial rehearsing of the stimuli. The developmental changes may occur at somewhat older CA levels in retardates than in normal children, but if they occur the course of development of short-term memory in retardates would be similar to that in normal children. Furthermore, there would be implications for the development of training techniques in memory skills for retarded as well as young normal children.

\section{EXPERIMENT I}

In the first experiment, the Label versus No Label conditions of the Hagen-Kingsley study (1968) were administered to mildly retarded children. The subjects were at an MA level at which labeling had been found to facilitate overall recall, but at a CA level where overall recall was not affected by labeling even though primacy and recency performance were.

Subjects

The subjects were residents of the Wayne County Child Development Center, Northville, MI. Of the total sample of 50, 39 were males and 11 were females. Half were assigned to the Label Group and half to the No Label Group. For the Label Group, 19 were males and 6 were females; for the No Label Group, 20 were males and 5 were females. Mean MA, $\mathrm{CA}$, and IQ levels for each of the groups were as follows: Label Group, $\mathrm{MA}=7.9$ years, $\mathrm{CA}=10.6$ years, and IQ (Stanford-Binet) $=74.5$; No Label Group, $\mathrm{MA}=7.8$ years, $\mathrm{CA}=10.6$ years, and $\mathrm{IQ}=73.5$.

\section{Materials and Procedure}

The materials and procedure were identical to those described in Hagen and Kingsley (1968), except that seven rather than eight pictures per trial were shown since pretesting indicated these subjects had difficulty with the longer series. Each stimulus card depicted a familiar animal. The seven cards were laid out one at a time in a row before the subject. Each was shown for approximately 2 sec. After all seven had been shown and placed face down in the row, a cue card, identical to one of the seven presentation cards, was shown and the child's task was to point to the face-down card in the row which matched the cue card. Order of presentation of the trials was randomized across subjects. A given picture was randomly placed in the series on a given trial so no 
learning of position was involved and each of the seven serial positions was probed once. In the Label condition, the subjects were told to say aloud the name of each picture as it was shown by the experimenter. In the No Label condition, the subjects were simply told to try to remember the locations of the pictures.

\section{RESULTS}

The proportion of correct responses constituted the overall memory measure. Overall performance for the two groups, Label versus No Label, did not differ $(t<1)$. The proportions of correct responses by serial position are presented in Fig. 1. The general shapes of these curves are similar to those obtained with normal subjects of similar MA (Hagen \& Kingsley, 1968). At the first, or primacy, position performance was elevated. However, unlike the performance of normal 10-year-old subjects, it declined at the second position. At the seventh, or recency, position performance was also elcvated. Since fewer serial positions were used in this study than the previous study, direct comparisons between the two cannot be made. It is evident, though, that the effect of labeling on performance of these retarded subjects was similar to that of the normal subjects; at the primacy portion of the curves, labeling resulted in lower performance, while at the recency portion, labeling improved performance. However, Hagen and Kingsley found labeling influenced two or three serial positions at recency, while for the retardates labeling facilitated only the most recent position.

In order to examine age trends in serial recall performance of the

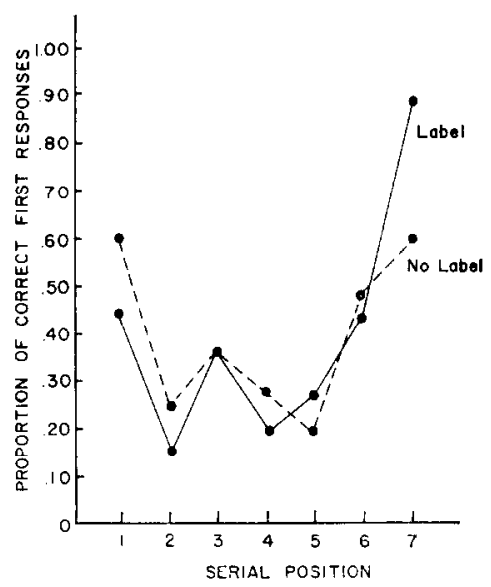

Fig. 1. Proportion of correct responses as a function of serial position for the Label and No Label Groups. 


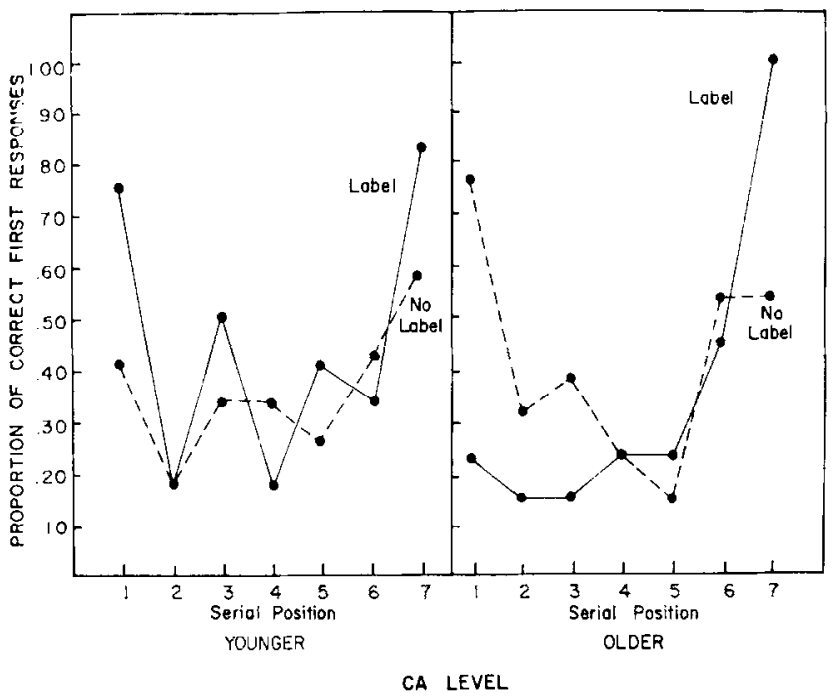

FIg. 2. Proportion of correct responses as a function of serial position for the Label and No Label Groups at the low- and high-CA levels.

retarded subjects, each group was divided into a low- and a high-CA group. The serial-position recall performance is shown in Fig. 2. For the low-CA group (mean $\mathrm{CA}=9.5$ years, Range $=8.5-10.6$ years, $N=24$ ), there was no clear effect due to labeling, while for the high-CA group (mean $\mathrm{CA}=11.2$ years, Range $=10.8-11.8$ years, $N=26$ ), the Label versus No Label effects were very similar to those found with normal 10 year olds (Hagen \& Kingsley, 1968). A three-way analysis of variance for low- versus high-CA level, Label versus No Label conditions, and serial positions (repeated measure) was performed. The only significant main effect was for serial positions $[F(6,276)=11.43, p<.01]$, and the interaction among CA level, experimental conditions, and serial position was also significant $[F(6,276)=2.86, p<.01]$. The labeling effect at the first serial position is especially striking in that labeling facilitated performance for the low-CA group but impeded performance for the high-CA group.

Since the serial recall data for the high-CA group were consistent with previous findings, additional analyses were performed on this group only. Overall, labeling did not have an effect (Label condition: mean proportion correct $=.35$; No Label condition: mean proportion correct $=.42$; $F<1.0$ ). At the first, or primacy, position labeling hindered recall $(t(24)=3.42, p<.01)$. At the seventh, or recency, position labeling facilitated recall $(t(24)=3.29, p<.01)$. It appears that labeling resulted in very similar changes in recall for these retarded children with 
an average CA of just over 11 years, as found in normal children with an average $\mathrm{CA}$ just over 10 years.

While comparisons of overall memory performance cannot be made directly since the task used in this study was somewhat easier than that used in the previous study, it is evident that overt verbal labeling affects serial position recall similarly in both normal and mildly retarded children and that both groups show similar developmental changes.

\section{EXPERIMENT II}

The findings of these studies (Hagen \& Kingsley, 1968, as well as Expt I) suggest that with increasing CA verbal rehearsal of the names of the to-be-remembered pictures increases, thereby improving recall, especially at those positions at the primacy portion of the recall curve. Further, in the Kingsley-Hagen study (1969) induced rehearsal facilitated recall at primacy positions for young children. It appears that these young children did not spontaneously rehearse and that supplying them with a rehearsal strategy facilitated recall. In addition, the experimenter not only taught the children to rehearse the series of names, but he also prompted them when they either rehearsed an item out of sequence or forgot a particular item. Prompting was seldom needed for the first two or three positions but was more frequently needed for the last positions. How important a role did this prompting play in facilitating recall? Would mildly retarded children benefit from induced rehearsal as did younger normal children? In order to answer these questions, this experiment used a sample of the subjects from the first experiment. There were three experimental conditions: (a) The subjects were required to rehearse out loud the names of pictures and prompting was provided when errors occurred (Prompt); (b) rehearsal was required but no prompting was provided (No Prompt); and (c) rehearsal was required and prompting was provided for the first three pictures (i.e., primacy positions) presented only (Primacy Prompt). This third condition was included as an attempt to simulate the rehearsal effects in normal children at this age level. By this age, improvement in recall occurs because of improved primacy recall, and the available evidence suggests that this improvement results from the use of spontaneous rehearsal. It was expected that this Primacy Prompt condition would produce serial recall similar to that found in normal children of the equivalent $\mathrm{CA}$ level.

\section{METHOD}

\section{Subjects}

A total of 36 subjects from Expt I, 29 males and 7 females, were included in this study. In the Prompt condition, there were 8 males and 
4 females; in the No Prompt condition, there were 10 males and 2 females; and in the Primacy Prompt condition, there were 11 males and 1 female. Mean MA, CA, and IQ levels for each of the conditions were as follows: Prompt: $\mathrm{MA}=7.8$ years, $\mathrm{CA}=10.7$ years, and $\mathrm{IQ}=74.2 ;$ No Prompt: $\mathrm{MA}=7.8$ years, $\mathrm{CA}=11.1$ years, and $\mathrm{IQ}=71.0$; and Primacy Prompt: $\mathrm{MA}=7.9$ years, $\mathrm{CA}=10.6$ years, and $\mathrm{IQ}=74.5$.

The subjects were chosen from those still available of the children tested in the first experiment and were then assigned randomly to the three experimental conditions. Testing occurred approximately three months after the completion of Expt I.

\section{Materials and Procedure}

The materials and procedure for the task were the same as they were in the first experiment, except that instead of the Label-versus-No Label instructions, there were now three sets of instructions for the three conditions. In the Prompt condition the child was told to say aloud the names of all the pictures in order as each new picture was presented, and that the experimenter would help him if he forgot any of the names by saying the correct names. This procedure is described more fully in Kingsley and Hagen (1969). For the No Prompt condition, the instructions were similar except no mention was made of prompting by the experimenter and no prompting occurred during the test trials. For the Primacy Prompt condition, the instructions were the same as for the first condition, except the subject was told the experimenter would help him if he forgot some of the names. Prompting was given for the names of the first three pictures and for only the first three rehearsal trials; hence prompting occurred only for the positions nearest the primacy portion of the serial position list. For all conditions two practice trials preceded the test trials.

In those cases where prompting occurred, the experimenter said only those names which had been said incorrectly by the subject, who then repeated the correct sequence of names.

\section{RESULTS}

The proportion of correct responses constituted the memory measure. A two-way analysis of variance compared effects among the three experimental conditions and serial positions (repeated measure). The differences among the conditions were not significant $(F(2,33)=2.34)$; but the serial position effect $(F(6,198)=10.70, p<.01)$ was significant. The interaction of conditions by serial position did not reach significance $(F(12,198)=1.74, p>.10)$. Figure 3 illustrates the findings for the three conditions at each of the serial positions of the recall task. It is evident from this figure that the results of the experimental manipulations 


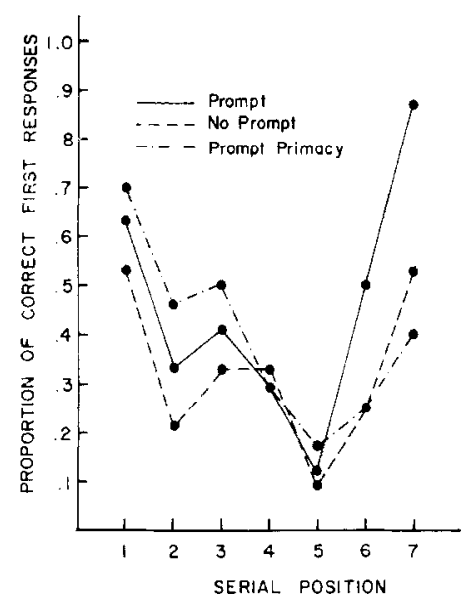

FIa. 3. Proportion of correct responses as a function of serial position for the Prompt, No Prompt, and Prompt Primacy Groups.

were in the expected directions. Since there were predictions concerning recall at serial positions 1-3 based on previous findings (Kingsley \& Hagen, 1969), the following analyses were performed.

Proportions of correct responses for the first three serial positions were compared for the three conditions, and the differences among them were not quite significant $(F(2,33)=2.52, p<.10)$. These recall scores for the Primacy Prompt condition, which received prompting for these three serial positions only, were higher than recall scores for the No Prompt condition $(t(22)=2.98, p<.01)$. Recall scores for the Prompt condition were higher, but not quite significantly so, than recall scores for the No Prompt condition $(t(22)=1.65, p<.10)$. Thus prompting had a discernible effect under some conditions.

Recall performance for the last, or most recent, positions for each of the three conditions was found to differ $(F(2,33)=13.06, p<.01)$. Recency recall for the Prompt condition was higher than the recall for either the No Prompt condition $(t(22)=2.29, p<.05)$ or the Primacy Prompt condition $(t(22)=4.55, p<.01)$. It is clear that when prompting was provided for the most recently presented items recall was improved. This finding replicated the finding from the Kingsley-Hagen study (1969) for young, normal children. These studies together show that either simple verbal labeling or induced rehearsal with prompting facilitate recency recall.

The findings of this experiment demonstrate that, even when no overall recall differences occur among conditions that vary rehearsal of to-be-recalled information, serial position analyses reveal differences in recall. 


\section{DISCUSSION}

These two experiments have demonstrated that serial position recall of mildly retarded children is in many respects similar to recall in normal children of approximately the same CA level. Verbal labeling facilitated total recall for normal 6- to 8-year-olds (Hagen \& Kingsley, 1968), but no overall facilitation was found either for normal or for retarded children just over 10 years of age (Expt I). For these children, labeling did affect serial position recall similarly. When the retarded sample was divided into younger versus older age levels, recall at the primacy portion was hindered by labeling for the older group only, replicating the finding for normal 10-year-old children. While a direct comparison of absolute memory levels cannot be made, since the task of the retardates was slightly easier than the task of the normal children, the retardates did somewhat less well: overall they recalled $40 \%$ correctly while the normal children recalled $46 \%$ of the pictures correctly. It is suggested by these findings that, while some differences may occur in total recall, the effects of labeling on serial position recall do not differ for retarded as compared to normal children.

Although induced verbal rehearsal had been found to facilitate recall in normal 5-year-old children (Kingsley \& Hagen, 1969) it was not known what role was played by the verbal prompting supplied by the experimenter. Nor was it known whether retarded children, who recalled less items correctly at the primacy positions than normal children of similar CA, would benefit from induced verbal rehearsal. The findings of Expt II indicate that the prompting provided by the experimenter in the induced rehearsal condition affected recall. When prompting was provided for all serial positions, a facilitation of recall at recency and a lesser effect at the primacy positions was found. When prompting was provided for only the primacy (first three) serial positions, recall at these positions was improved. These findings are consistent with the argument that improvement in primacy recall comes about through the use of "rehearsal strategies." When these strategies are used spontaneously, i.e., they are not required by an experimental manipulation, they evidently entail some sort of self-prompting which operates similarly to the prompting provided by the experimenter in the prompt conditions. Older subjects may attempt to rehearse only the items at the primacy positions when they realize it is not possible to rehearse all items with a high degree of accuracy.

Whether retarded children exhibit actual differences or developmental lags in cognitive abilities when compared to normal children has been the subject of considerable research and debate (Zigler, 1969). The view that young, normal children perform less well in short-term memory 
because of a production deficiency rather than a mediational deficiency had been argued convincingly by Flavell (1970), and this view also has relevance to our understanding of the differences found in recall of retardates in the studies reported here as well as elsewhere (e.g., Ellis, 1970). The results of Expt I are consistent with the view that required labeling of information to be recalled facilitates recall for those subjects who do not spontaneously produce appropriate mnemonics during presentation of stimuli but interferes with recall of primacy items for those subjects who do use mnemonics. The results are similar for retarded (Expt I) and normal children (Hagen \& Kingsley, 1968). In Expt II, evidence was obtained that suggests retardates to profit from induced rehearsal as do normal children (Kingsley \& Hagen, 1969). Just why younger and retarded children do not use rehearsal strategies spontaneously is still open to question. However, it is apparent that they can do so under certain circumstances, so it does not seem likely that they have a cognitive deficit that impairs their memory ability. Whether training in the use of rehearsal strategies can result in generalized gains in memory and improvement in future memory tasks remains to be determined through subsequent research.

\section{REFERENCES}

Belmont, J. M., \& ButTerfield, E. C. What the development of short-term memory is. Human Development, 1971, 14, 236-248.

Eldis, N. The stimulus trace and behavioral inadequacy. In N. Ellis (Ed.), Handbook of mental deficiency. New York: MoGraw-Hill, 1963.

EldIs, N. R. Memory processes in retardates and normals. In N. R. Ellis (Ed.), International review of research in mental retardation, Vol. 4. New York: Academic Press, 1970.

Flavell, J. H. Levelopmental studies of mediated memory. In H. W. Reese \& L. P. Lipsitt (Eds.), Advances in child development and behavior, Vol. 5. New York: Academic Press, 1970.

Flavell, J. H. What is memory development the development of? Human Development, 1971, 14, 272-278.

Hagen, J. W., Meacham, J. A., \& Mesibov, G. Verbal labeling, rehearsal, and shortterm memory. Cognitive Psychology, 1970, 1, 47-58.

Hagen, J. W., \& Kingsley, P. R. Labeling effects in short-term memory. Child Development, 1968, 39, 113-121.

Kenney, T. J., Cannizzo, S. R., \& Fravelu, J. H. Spontaneous and induced verbal rehearsal in a recall task. Child Development, 1967, 38, 953-966.

Kingsley, P. R., \& Hagen, J. W. Induced versus spontaneous rehearsal in shortterm memory in nursery school children. Developmental Psychology, 1969, 1, 40-46.

Ztgler, E. Developmental versus difference theorics of mental retardation and the problem of motivation. American Journal of Mental Deficiency, 1969, 73, 536-556. 